\title{
Scepter-Mini Balloon Assisted Coil Embolization of an Intracranial Arterial Aneurysm in a Child with PHACE Syndrome via a Persistent Trigeminal Artery
}

\author{
Bharathi Dasan Jagadeesan, MD, Coridon Quinn IV, MD, Kamran Masood, MB, BS, \\ Andrew Grande, MD, Ramachandra Prasad Tummala, MD \\ Depatment of Radiology, Neurosurgery and Neurology, University of Minnesota, Minneapolis, MN, USA
}

\begin{abstract}
Cerebral vascular malformations constitute one of the key abnormalities in children with PHACE syndrome, which is characterized by Plaque like cutaneous hemangiomas, Posterior fossa abnormalities, arterial Cerebrovascular and Eye abnormalities, with or without Sternal clefts (PHACES when sternal clefts are present), and associated midline anomalies. Both moyamoya arteriopathy and intracranial aneurysms have been reported in children with this syndrome. Herein, we report the successful treatment of a growing left posterior-communicating artery aneurysm arising from an aberrant left internal carotid artery (LICA) with balloon assisted coiling (BAC) in a child with PHACE syndrome. We circumvented the limitations posed by the narrow caliber of the proximal LICA, by successfully navigating a coiling microcatheter from the basilar artery into the LICA via a persistent trigeminal artery. BAC was then achieved using a Scepter Mini balloon microcatheter for aneurysm neck remodelling.
\end{abstract}

Key Words: PHACE syndrome; Aneurysm; Persistent trigeminal artery; Coil embolization; Scepter mini balloon

\section{INTRODUCTION}

This report illustrates the endovascular management of a growing brain aneurysm in a child with PHACE syndrome (OMIM 606519), a rare non-inherited congenital neurocutaneous syndrome with unknown etiopathogenesis wherein cerebrovascular malformations are the most common extra-cutaneous abnormalities. ${ }^{1-3}$ Endovascular management of brain aneurysms in young children can be challenging due to small parent vessel diameters, non-availability of specialized low profile microcatheters, and relative contraindications to stent or flow diverter placement due to the changes that are anticipated with the child's growth. ${ }^{4,5}$ These challenges are further exacerbated when intracranial aneurysms occur in the setting of syndromic cerebrovascular abnormalities such as PHACE. It is hoped that perusal of this report will (1) enhance the familiarity of readers with PHACE syndrome, (2) highlight the adaptation of the novel low-profile SMB to perform balloon assisted coiling (BAC) in a child, and (3) demonstrate the feasibility of utilizing the persistent trigeminal artery (PTA)

\section{Correspondence to:} Bharathi Dasan Jagadeesan, MD Depatment of Radiology, Neurosurgery and Neurology, University of Minnesota, MMC 292, Mayo Memorial Building, 420 Delaware St SE, Minneapolis, MN 55455, USA

Tel: $+1-6126265566$

Fax: +1-6126265505

E-mail: jagad002@umn.edu

Received: February 4, 2021 Revised: April 29, 2021 Accepted: June 17, 2021

Copyright $\odot 2021$ Korean Society of Interventional Neuroradiology This is an Open Access article distributed under the terms of the Creative Commons Attribution Non-Commercial License (http://creativecommons.org/licenses/by-nc/4.0) which permits unrestricted non-commercial use, distribution, and reproduction in any medium, provided the original work is properly cited.

pISSN 2093-9043 eISSN 2233-6273 
to gain internal carotid artery aneurysm sac access from a posterior circulation approach.

\section{CASE REPORT}

The patient had initially presented to our center as an infant for the management of multiple cerebrovascular abnormalities that had been discovered on a computed tomography angiogram, and digital subtraction angiogram (DSA) of the head that had been performed at an outside center as part of the work up for PHACE syndrome. That work up had been initiated by the presence of a progressing facial hemangioma, left eye ptosis, and a cardiac murmur. These initial imaging studies had revealed persistent fetal circulatory patterns in the form of an aberrant left internal carotid artery (which also gave rise to a prominent ascending pharyngeal branch) with a narrow cervical segment and postero-lateral course at the skull base, and a prominent PTA between the cavernous segment of the left internal carotid artery (LICA) and the mid-basilar artery. In addition, the more superior segments of the LICA were dysplastic with segmental narrowing and dilatation, and the entire right ICA, (which terminated at the communicating segment) was also extremely dysplastic with a tortuous corkscrew course. The upper basilar artery was significantly narrowed with multiple moyamoya like perforating arteries arising from the top of the basilar artery as well as the terminal regions of the internal carotid arteries. The right anterior cerebral artery and middle cerebral artery were exclusively supplied from the LICA via the Anterior communicating artery.

Bilateral posterior communicating artery (PCoA) segment aneurysms were noted, as well as a top of basilar aneurysm and very dysplastic aneurysmal regions at the junction of the right PCOA and the right posterior cerebral artery (Fig. 1A). These malformations were observed with serial magnetic resonance angiographic (MRA) imaging, performed at 6, 12, and 24 months following the initial imaging studies. A significant enlargement of the left posterior communicating artery aneurysm (PCOM) aneurysm from $4 \times 5 \times 4 \mathrm{~mm}$ to $7 \times 5 \times 8 \mathrm{~mm}$ was reported on cross sectional MRA between the second and third follow up studies mentioned above. We then decided to opt for endovascular treatment of this growing left PCOA aneurysm. This decision was based upon the increased risk of rupture posed by this growing aneurysm, the relatively higher operative risk associated with surgical clipping in the setting of multiple vascular malformations and variations, and our experience with endovascular management of cerebrovascular pathology in the patient's age group. In terms of endovascular treatment paradigms, although flow diversion was considered, the narrowing of the proximal ICA, as well as the anticipated need for future surgical bypass procedures (for moyamoya like changes) resulted in not proceeding with this option. ${ }^{6-8}$ Likewise, it was thought best to avoid stent placement within the intracranial arteries.

\section{Treatment}

Bilateral common femoral arterial access was obtained under ultrasound guidance. Anticoagulation was obtained with the intrvaneous administration of 100 units of Heparin per kilogram body weight. We then selected the left common carotid artery using a 4 French, $65 \mathrm{~cm}$ angle glide catheter advanced through a 4 French arterial sheath placed in the left common femoral artery. The left vertebral artery was selected via a right groin access with a 5 French, $115 \mathrm{~cm}$ Sofia intermediate catheter (Microvention, Aliso Viejo, CA, USA). Simultaneous contrast injections were performed through both catheters, initially for diagnostic DSA; and thereafter to obtain digital road maps that delineated the LICA, the left vertebral artery and basilar artery clearly. The left PCoA aneurysm was noted to be $11.2 \times 7 \times 6 \mathrm{~mm}$ in size, it had a complex shape and a wide neck ( $6 \mathrm{~mm})$. We then advanced an Echelon 10 (Medtronic, Irvine, CA, USA) over a Synchro soft microwire (Stryker Neurovascular, Fremont, CA, USA) through the Sofia catheter into the basilar artery and the PTA was selected. The Echelon 10 microcatheter was then very carefully advanced across the PTA to safely catheterize the LICA (Fig. 1B-D). Subsequently, we prepared a Scepter-mini balloon microcatheter (Microvention) over an Asahi 0.008 inch microwire (Medtronic) without ex-vivo pre-inflation as described elsewhere. ${ }^{9}$ The balloon microcatheter was then advanced through the angle glide catheter into the narrow segment of the proximal LICA and eventually into the intracranial ICA such that the balloon markers were across the neck of the PCOM aneurysm (Fig. 1E-G). The diameter of the LICA at this site was $1.9 \mathrm{~mm}$, and the Scepter mini balloon has a nominal diameter of $2.5 \mathrm{~mm}$, and length of $9 \mathrm{~mm}$. Subsequently, Multiple hydrogel coated platinum coils were then placed into the aneurysm sac through the Echelon 10 microcatheter, while the aneurysm neck was remodelled with inflation of the Scepter mini balloon, and complete obliteration of the aneurysm was achieved 

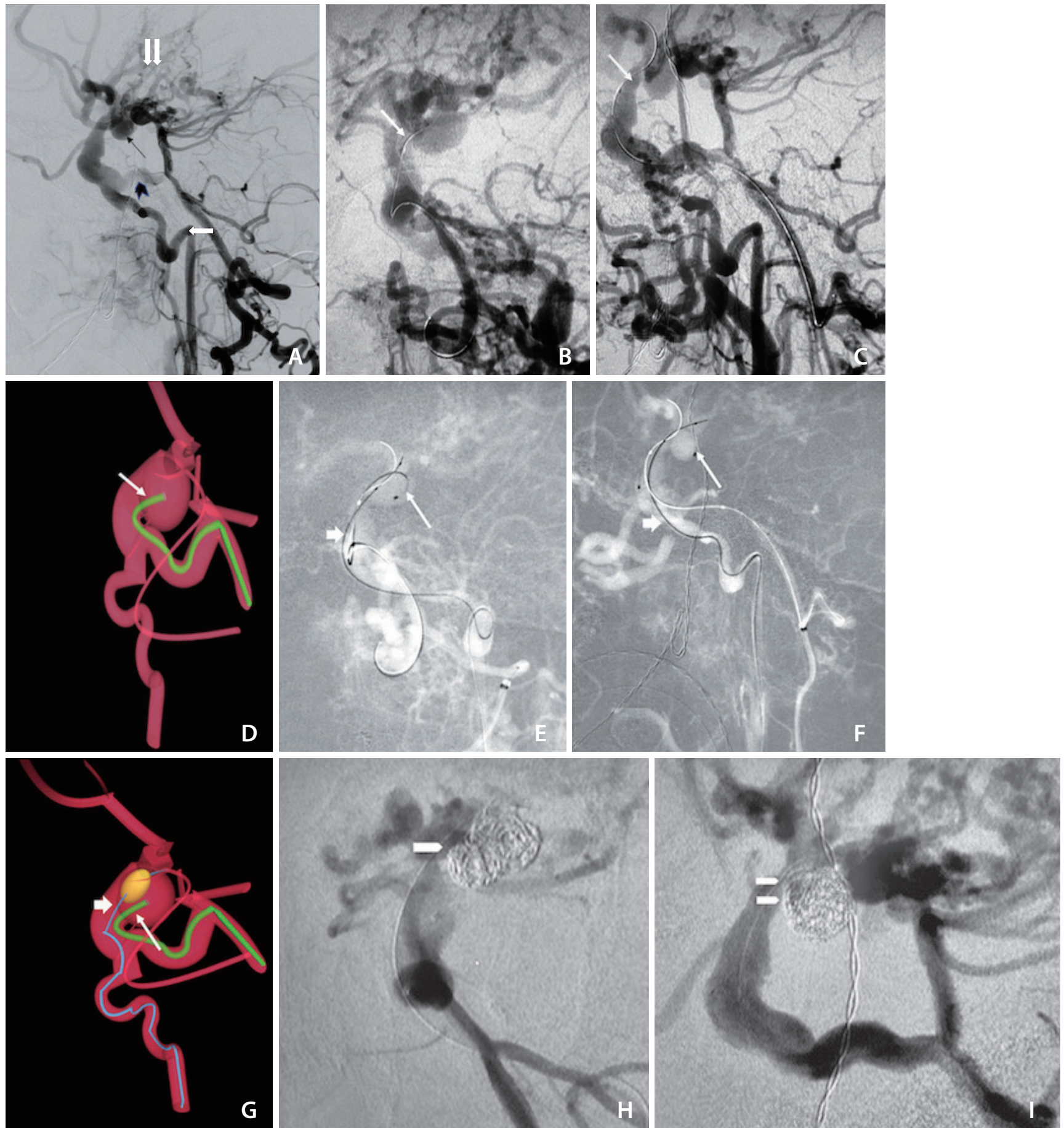

Fig. 1. (A) Lateral projection from DSA obtained with simultaneous injection of the LICA and left vertebral artery showing 1. PTA (black chevron), 2. Aberrant LICA (single white arrow), 3. left PCoA aneurysm (single black arrow) and 4. Moyamoya like changes (double white arrow). (B) Anterior and (C) lateral oblique projections showing an Echelon 10 microcatheter (white arrows) advanced from the basilar artery to the LICA via the PTA, and (D) illustration showing Echelon 10 microcatheter (green line/white arrow) advanced from the basilar artery to the LICA via the PTA. (E) Anterior and (F) lateral oblique projections showing an Echelon 10 microcatheter (thin white arrows) advanced from the basilar artery to the LICA via the PTA, and a SMB advanced (thick white arrows) advanced across the neck of the PCoA aneurysm, and (G) illustration showing relative positioning of Echelon 10 microcatheter (thin white arrow) and SMB (thick white arrow). (H) Anteroposterior and (I) lateral DSA projections showing obliteration of the PCoA aneurysm by a coil mass (white arrows) with preserved flow in the parent vessel. DSA, digital subtraction angiogram; LICA, left internal carotid artery; PTA, persistent trigeminal artery; PCoA, posterior-communicating artery; SMB, Scepter Mini balloon microcatheter. 
(Fig. 1H, I). A total of $35 \mathrm{~mL}$ of lodinated Contrast was used for the procedure.

\section{Outcome and follow up}

Complete obliteration of the aneurysm was achieved with no intra or post procedural complications. The child was discharged home the next day and continues to do well at 90 days post-procedure.

\section{DISCUSSION}

Our report illustrates some of the common cerebrovascular malformations found in children with PHACE syndrome as well as a trans PTA approach for performing balloon assisted aneurysm coiling in a child with this syndrome. PHACE syndrome has a low prevalence in the general population $(<1: 1,000,000)$. However, it is not infrequent for cerebrovascular specialists working in large pediatric centers to encounter children affected by this disorder. Such children, who often present for evaluation of cutaneous malformations are eventually diagnosed with PHACE syndrome on further workup by a multidisciplinary pediatric vascular clinic. The cerebrovascular malformations that can be found in these children are those illustrated here, such as persistent primitive embryonic arteries, agenesis or stenosis of the internal carotid and vertebral arteries, moyamoya disease like intracranial arterial changes; and fusiform and saccular intracranial aneurysms., ${ }^{1,10}$

The SMB was considered to be the best choice for BAC in this child due to its low profile (which enabled us to advance it through a narrowed segment of the ICA in an atraumatic fashion, while using just a 4 French angled glide diagnostic catheter for support), as opposed to other balloon microcatheters, such as Scepter C or XC; Hyperform/Hyperglide (Medtronic), Transform (Stryker Neurovascular) etc., that could also have been used, but have larger inflation profiles. The SMB also has a maximum inflation diameter of $2.7 \mathrm{~mm}$, that mitigates against inadvertent balloon overinflation within the small caliber intracranial arteries found in children. Similarly, the Echelon 10 microcatheter (Medtronic) was utilized due to its low profile as well as its unique torqueability which allowed for the catheterization of the aneurysm at a difficult backwards angle.

\section{Fund}

None.

\section{Ethics Statement}

The authors' institutional IRB waives approval for case reports with less than 2 cases. Since the consent for publication was not available for the patient in this case report, the patient's information was anonymized by removing the sex and specific age.

\section{Conflicts of Interest}

The authors have no conflicts to disclose.

\section{Author Contributions}

Concept and design: BJ and RT. Analysis and interpretation: BJ and RT. Data collection: BJ, CQIV, and KM. Writing the article: BJ. Critical revision of the article: CQIV, KM, AG, and RT. Final approval of the article: BJ. Overall responsibility: BJ.

\section{ORCID}

Bharathi Dasan Jagadeesan:

https://orcid.org/0000-0002-1143-941X

Coridon Quinn IV: https://orcid.org/0000-0002-1064-2838

Kamran Masood: https://orcid.org/0000-0003-4494-4241

Andrew Grande: https://orcid.org/0000-0002-6474-7969

Ramachandra Prasad Tummala:

https://orcid.org/0000-0002-1905-2906

\section{REFERENCES}

1. Heyer GL, Dowling MM, Licht DJ, Tay SK, Morel K, Garzon MC, et al. The cerebral vasculopathy of PHACES syndrome. Stroke 2008;39:308-316

2. Frieden IJ, Reese V, Cohen D. PHACE syndrome. The association of posterior fossa brain malformations, hemangiomas, arterial anomalies, coarctation of the aorta and cardiac defects, and eye abnormalities. Arch Dermatol 1996;132:307-311

3. Hadisurya J, Guey S, Grangeon L, Wieczorek D, Corpechot M, Schwitalla JC, et al. Moyamoya angiopathy in PHACE syndrome not associated with RNF213 variants. Childs Nerv Syst 2019;35:1231-1237

4. Lv X, Jiang C, Li Y, Yang X, Wu Z. Endovascular treatment for pediatric intracranial aneurysms. Neuroradiology 2009;51:749-754

5. Ghali MGZ, Srinivasan VM, Cherian J, Wagner KM, Chen SR, Johnson J, et al. Multimodal treatment of intracranial aneurysms in children: clinical case series and review of the literature. World Neurosurg 2018;111:e294-e307

6. Jernigan S, Storey A, Hammer C, Riordan C, Orbach DB, Scott 
RM, et al. Moyamoya syndrome and PHACE syndrome: clinical and radiographic characterization of the intracranial arteriopathy and response to surgical revascularization. J Neurosurg Pediatr 2019;23:493-497

7. Heyer GL, Millar WS, Ghatan S, Garzon MC. The neurologic aspects of PHACE: case report and review of the literature. Pediatr Neuro/ 2006;35:419-424

8. Habib M, Manilha R, Khan N. Management of PHACES syndrome: risk of stroke and its prevention from a neurosurgical perspective. Eur J Paediatr Neurol 2020;26:39-45

9. Mehta T, Hassan A, Masood K, Tekle W, Grande A, Tummala R, et al. The next step in balloon assisted endovascular neurosurgical procedures: A case series of initial experience with the Scepter Mini balloon microcatheter. Interv Neuroradio/ 2021;27:298-306

10. Oza VS, Wang E, Berenstein A, Waner M, Lefton D, Wells J, et al. PHACES association: a neuroradiologic review of 17 patients. AJNR Am J Neuroradiol 2008;29:807-813 